\title{
INOVAÇÃO PARA A SUSTENTABILIDADE
}

\section{Fernando Galembeck}

Laboratório Nacional de Nanotecnologia do Centro de Pesquisa em Energia e Materiais / Instituto de Química, Universidade Estadual de Campinas, 13083-970 Campinas - SP, Brasil

Recebido em 31/7/13; aceito em 7/10/13; publicado na web em 24/10/13

INNOVATION FOR SUSTAINABILITY. The transition to sustainable standards of production and consumption within a scenario of decreased availability of natural resources, growing population and climate change is essential to meet current challenges facing mankind. A strategy to meet these challenges should contain elements different from current approaches for industrial production. This work concentrates on the possibilities for the intensive use of sustainable biomass, abundant minerals and every type of residue, a task that can largely benefit from the application of nanotechnology and biotechnology platforms for material design and transformation. This strategy cannot be solely based on existing knowledge and requires new science, new knowledge including supposedly wellknown themes, like the tribochemistry of electrostatic charging and friction. It is especially relevant within the Brazilian context, where many recent successful innovations are related to biomass production and transformation. Implementation of this strategy requires converging efforts by personnel from many different organizations and professions, while making sound risk assessment to produce significant innovation leading to sustainable development.

Keywords: innovation; sustainability; nanotechnology.

\section{INTRODUÇÃO}

A produção de respostas aos grandes desafios atuais e futuros do planeta e da humanidade (educação, cidadania plena, mudança climática, produção e qualidade dos alimentos, acesso e qualidade da água, segurança energética, preservação de ecossistemas e das espécies, doenças emergentes e qualidade de vida) ${ }^{1}$ exige a construção de padrões sustentáveis de produção e consumo. Sem isso, não será possível atender às crescentes demandas por melhor qualidade de vida, feitas por cada vez mais pessoas, em um cenário de muitas incertezas, mas com sinais de esgotamento ou aumentos dos custos de exploração dos recursos naturais, além das mudanças climáticas.

Nesse artigo, analisamos o contexto atual de disponibilidade de matérias-primas e de sua transformação, enfatizando três tipos de fontes: os minerais abundantes, a biomassa e os resíduos municipais, industriais e do agronegócio. Em seguida, tratamos dos papéis da nanotecnologia, biotecnologia e tecnologias de informação como plataformas de transformação de matérias-primas em bens de uso em processos de produção e também em bens de consumo. Finalmente, identificamos vantagens e desvantagens do contexto brasileiro, delineando algumas estratégias de inovação que permita a continuidade do desenvolvimento.

\section{A EXAUSTÃO DOS RECURSOS NATURAIS}

Há algumas décadas, alguns estudiosos e especialmente o Clube de Roma ${ }^{2}$ vem publicando estudos mostrando que a utilização crescente e intensiva de reservas minerais gera cenários de escassez de recursos naturais no futuro próximo. A falta de substâncias essenciais à produção implica em escassez de alimentos e de matérias-primas industriais, inviabilizando o crescimento econômico e gerando más perspectivas de qualidade de vida para a maioria da população. Por outro lado, muitas pessoas minimizam a importância dessas análises classificando-as de neomalthusianas e lembrando que a história da humanidade posterior a Malthus não confirmou suas apreensões. Por

*e-mail: fernando.galembeck@1nnano.cnpem.br exemplo, a "revolução verde", fruto da tecnologia e especialmente da produção em grande escala de fertilizantes é frequentemente apontada como sendo um claro desmentido de Malthus, e a recente introdução do gás de xisto no cenário global de energia é apontada por alguns como sendo um desmentido dos cenários negativos de disponibilidade de combustíveis fósseis.

Entretanto, muitos dados sobre a futura disponibilidade de recursos naturais são realmente preocupantes e um exemplo nítido é o das rochas fosfáticas intensamente utilizadas na produção de fertilizantes consumidos na produção de alimentos, energia e matérias-primas derivadas da biomassa.

Não existe substituto para o fósforo na agricultura e as rochas fosfáticas são a principal fonte desse elemento. Dados oficiais de 2007 mostram que o Brasil conta com reservas de 319 milhões de toneladas destas rochas, que são exploradas principalmente pela Vale em minas existentes em SP, MG, GO e BA. Embora as reservas de fosfato sejam suficientes para garantir o atual consumo interno por mais 30 anos, a produção interna não cresce no mesmo ritmo da demanda: em 2007 o Brasil produziu 6,2 milhões de toneladas de fosfato, mas neste mesmo ano $50 \%$ do consumo brasileiro foi suprido por importações, devido ao crescimento de $8,8 \%$ na demanda do concentrado de rocha para fertilizantes e de 4,5\% na demanda de acido fosfórico. Fosfatos também são usados nas indústrias de refrigerantes, de detergentes e de ração animal. Nestes segmentos, a demanda de fosfatos foi ainda maior, crescendo $33,3 \%$ no mesmo período. ${ }^{3}$

Globalmente, somente dois países no mundo disporiam de reservas de fosfatos suficientes para manter o atual nível de utilização, por alguns séculos: China e Marrocos. Por outro lado, em 2010 o International Fertilizer Development Center (IFDC) determinou que as reservas de rocha fosfática são suficientes por vários séculos e, em 2011, o U.S. Geological Survey refez sua estimativa de reservas, de 17,63 para 71,65 bilhões de toneladas, de acordo com o IFDC. Além disso, há evidências de que a quantidade de fósforo sendo trazida para a crosta pelos movimentos tectônicos seja equivalente à quantidade de rocha fosfática que está sendo extraída, hoje. ${ }^{4}$ Portanto, o problema não é exatamente o da existência ou não de fósforo, mas o da existência de conhecimento para a sua extração e aproveitamento, não apenas 
em escala global, mas também em escala nacional e mesmo regional.

De qualquer forma, a atual posição brasileira é desconfortável e as demandas crescentes só fazem piorar o quadro atual, exigindo novas soluções.

Situações análogas à do fósforo ocorrem com muitos outros elementos utilizados na produção industrial e agrícola, minuciosamente examinados em uma recente publicação da Royal Society of Chemistry inglesa. ${ }^{5}$ Entretanto, muitos mecanismos podem ser e estão sendo usados para enfrentar situações indesejáveis, construindo cenários mais positivos.

\section{ALTERNATIVAS AO QUADRO ATUAL}

Os desenvolvimentos científicos e tecnológicos recentes têm criado um grande número de oportunidades de criação de novos materiais avançados produzidos a partir de matérias primas obtidas de fontes renováveis, biomassa e lixo, e de minerais abundantes. Estas oportunidades respondem positivamente às necessidades de transição para uma economia caracterizada pelo desenvolvimento sustentável e de baixo carbono, que contribua para a mitigação das emissões e, portanto, para a minimização das contribuições antrópicas às mudanças climáticas globais. Propostas de desenvolvimento de materiais avançados, agregadoras de valor a produtos e resíduos de cadeias produtivas vigorosas no Brasil e baseadas em matérias primas abundantes ou renováveis foram anteriormente apresentadas em um estudo realizado para o Centro de Gestão e Estudos Estratégicos (CGEE),${ }^{6}$ abordando os seguintes tópicos:

1) Caracterização de materiais avançados e de suas fontes naturais;

2) Rotas alternativas para produção de insumos básicos para fertilizantes;

3) Produção de materiais agroquímicos avançados;

4) Reaproveitamento de rejeitos da atividade mineral e do agronegócio como insumos para produção de materiais avançados;

5) Produção de materiais avançados a partir de óleos e gorduras;

6) Produção de materiais avançados a partir de argilas;

7) Produção de materiais avançados a partir de fibras naturais;

8) Produção de materiais avançados a partir de borracha de látex natural.

Um fator de estímulo ao uso de matérias-primas derivadas da agricultura é a sua crescente disponibilidade, graças ao aumento na produção e uso de biomassa para a produção de alimentos e energia. Repete-se hoje no Brasil e em alguns outros países o que ocorreu com o petróleo nos países mais desenvolvidos, durante o século 20 , quando uma matéria-prima de combustíveis e energia tornou-se a fonte de milhões de toneladas de substâncias químicas (inclusive plásticos e borrachas, detergentes, amônia e ureia, hidrogênio) produzidos pela vasta indústria petroquímica com um grande potencial de satisfazer necessidades humanas. Da mesma forma, a produção de combustíveis a partir da agricultura, em grande escala e a preços vantajosos face aos das competidoras, já criou uma situação na qual a cana de açúcar e o milho são também fontes de plásticos e resinas.

Estes fatos demonstram a possibilidade da transição para uma economia de baixo consumo de petróleo e gás natural, desejável em qualquer estratégia de sobrevivência humana, em longo prazo. Por outro lado, torna-se importante para que se reduzam as emissões de carbono fóssil para a atmosfera. Mais importante ainda, a produção de biomassa é a única maneira hoje disponível de sequestrar carbono da atmosfera, sem qualquer gasto explícito de energia.

A produção de materiais avançados a partir de matérias-primas abundantes também resolve um problema global emergente, que é o aumento nas demandas de vastas populações humanas por melhores condições de vida. $\mathrm{O}$ atendimento dessas demandas dentro dos atuais padrões de consumo e de uso de materiais para a habitação, transportes, vestuário e segurança alimentar tem criado pressões imensas sobre recursos naturais não renováveis. Essas pressões podem e devem ser drasticamente reduzidas pelo uso de materiais renováveis ou muito abundantes na crosta terrestre, que está exemplificado neste artigo.

O sucesso no desenvolvimento de materiais a partir dos produtos agrícolas e minerais abundantes está criando respostas positivas para algumas das principais demandas coletivas da humanidade: melhoria na qualidade de vida, mitigação de emissões de gases que contribuem para o efeito estufa, redução nos descartes de efluentes sólidos e líquidos e outras formas de proteção do meio-ambiente. Alem disso, pode contribuir para o aumento na oferta de alimentos e energia. ${ }^{7}$

O momento atual oferece um grande número de novos conceitos e ferramentas experimentais para a exploração de muitas novas e antigas estratégias de desenvolvimento de materiais. Além disso, há também inúmeras novas possibilidades de criação biológica de novas matérias-primas e materiais, por meio da genômica e outras ômicas, bem como da nova disciplina da biologia sintética. Portanto, temos um cenário fértil para a inovação.

Uma característica das propostas de aproveitamento inovador da biomassa é o seu baixo risco de criação de impactos adversos sobre a saúde humana e sobre o meio ambiente. Este risco está sempre presente em qualquer nova tecnologia e nos respectivos produtos e em particular no desenvolvimento de materiais, tendo sido causa de muitos desastres com perdas humanas e econômicas vultosas. Há vários exemplos mais ou menos recentes da introdução de materiais que em certo momento pareceram dotados de propriedades maravilhosas, como o amianto, mas depois foram identificados como causadores de muitas mortes humanas e também da quebra de grandes empresas.

Matérias-primas derivadas da biomassa e de minerais abundantes são muito familiares à espécie humana, que hoje conta com séculos de experimentação no seu uso e de avaliação dos seus impactos. ${ }^{8}$ Isso não permite afirmar a ausência de risco no desenvolvimento de materiais avançados a partir destas matérias primas, uma vez que novas nanoestruturas poderão sempre produzir resultados inesperados. Entretanto, a experiência humana com argilas, por exemplo, é muito maior do que a relativa aos vários tipos de nanotubos, por exemplo.

Portanto, a criação e desenvolvimento de novos materiais avançados produzidos a partir de matérias primas obtidas de fontes renováveis e de minerais abundantes é um caminho aberto para a inovação em materiais no Brasil, integrador e viabilizador de políticas públicas, que pode ser empresarialmente atraente e tem grandes possibilidades de positivos impactos sociais e ambientais. Acima disso tudo, esse caminho está baseado em ciclos de vida que podem ser percorridos ciclicamente, o que é essencial para a sustentabilidade.

\section{Minerais abundantes}

A disponibilidade de um elemento depende da quantidade existente na crosta terrestre, do seu nível de concentração e da existência de tecnologias que tornem sua exploração economicamente viável e ambientalmente aceitável. Portanto, um caminho para a superação das limitações atuais na disponibilidade de elementos é o desenvolvimento de novas tecnologias de extração e refino de minerais.

Por outro lado, alguns insumos minerais básicos, como as argilas, areias, calcários, trona, siltes e vários tipos de rochas são realmente abundantes e familiares aos seres humanos, que por isso mesmo contam com séculos de experimentação no seu uso e na avaliação dos seus impactos. Isso não permite afirmar que sempre haverá ausência de risco no desenvolvimento de materiais avançados a partir destas matérias-primas, uma vez que novas nanoestruturas sempre poderão produzir resultados inesperados. Entretanto, a base histórica de conhecimento destes minerais é muito maior do que a que hoje existe sobre os materiais e partículas de criação mais 
recente. Em consequência, inovação de base tecnológica associada aos nanocompósitos poliméricos que utilizam argilas tem ocorrido mais rapidamente que no caso nanopartículas mais exóticas, criando novos estímulos à pesquisa sobre argilas naturais e seus análogos sintéticos.

\section{Biomassa}

Biomassa tem sido uma importante fonte de alimentos e de materiais para os seres humanos e mesmo para animais e aves, desde sempre. Sua importância relativa diminuiu com a emergência de materiais sintéticos, no século 20, mas ela é hoje fonte de matérias-primas de solventes "verdes" e de polímeros "petroquímicos" e a atividade de pesquisa em química verde está produzindo resultados importantes na substituição do petróleo por biomassa. O agronegócio gera grande quantidade de resíduos lignocelulósicos, graxos e isoprênicos cujo aproveitamento é geralmente reduzido, principalmente em produtos de alto valor agregado.

No passado, houve uma importante produção de filmes e fibras celulósicas, que foi depois drasticamente reduzida por razões ambientais, uma vez que os processos produtivos (xantogenato, cupramônio) geravam grandes quantidades de resíduos. Hoje, fibras de celulose regenerada são fabricadas a partir de soluções de celulose em dimetilformamida na presença de cloreto de lítio e outros solventes caros e exóticos, mas recicláveis, o que torna o processo economicamente viável.

Embora celulose seja uma das substâncias mais estudadas cientificamente, o conhecimento sobre ela ainda não recebeu contribuições de muitas técnicas de caracterização estrutural desenvolvidas nas últimas décadas especialmente as técnicas mais recentes de microscopia e tomografia eletrônica, bem como de microscopias de varredura por sondas. Apesar de a caracterização microestrutural e microquímica da biomassa lignocelulósica e de suas frações ser essencial para o design de novos materiais, este ainda se encontra em um estágio pouco avançado. Mesmo assim, o Brasil vem colecionando grandes sucessos tecnológicos e empresariais na produção de polpa e papel utilizando matérias-primas não clássicas, como a madeira de eucalipto e o bagaço de cana.

A incorporação física de partículas (argila, fibras, nanotubos) em matrizes poliméricas pode produzir materiais que apresentam vantagens com relação ao polímero virgem, nas suas propriedades mecânicas, térmicas, ópticas, de resistência à água e a solventes e como barreira de gases. ${ }^{9}$ Além disso, a adição de fibras naturais contribui para que os compósitos tornem-se ambientalmente corretos. ${ }^{10}$

É preciso intensificar as técnicas de microanálise por espectroscopia de energia dispersiva de raios- X (EDS) e espectroscopia por perda de energia (EELS), aliadas às técnicas modernas de microscopia de força atômica, que fornecem informações em um nível de detalhamento micro e nanométrico sobre diferentes frações lignocelulósicas, indo muito além do que pode ser atualmente observado na literatura científica e verificando dessa forma, a adequação desses materiais como fase dispersa em compósitos poliméricos.

$\mathrm{O}$ grupo do autor desse trabalho tem se aplicado à caracterização de partículas ${ }^{11}$ e de nanocompósitos ${ }^{12}$ utilizando diversas técnicas microscópicas. Recentemente, foi introduzida a técnica de mapeamento molecular baseada em microscopia eletrônica de transmissão associada à espectroscopia de perda de energia de elétrons (ESI-TEM), ${ }^{13}$ que tem sido muito útil no exame de sistemas polifásicos, com mais de um componente polimérico. Além disso, as microscopias de sonda em polímeros, especialmente as microscopias elétricas (KFM, EFM), permitiram demonstrar a importância da adesão eletrostática em sistemas polifásicos, evidenciando um novo mecanismo de adesão em sistemas multifásicos, a adesão eletrostática mediada por íons comuns $\left(\mathrm{Na}^{+}\right.$, etc. $)$
Muitos métodos são utilizados atualmente no fracionamento de biomassa, especialmente processos termoquímicos à base de ácidos, bases, água quente ou sob irradiação com microondas. ${ }^{14} \mathrm{O}$ controle dos diferentes processos de tratamentos produz uma ampla gama de estruturas, com tamanhos, formas, áreas e propriedades superficiais diferenciadas, o que deve permitir a preparação de compósitos e nanocompósitos com propriedades também diferenciadas.

\section{Resíduos}

O aproveitamento de resíduos é uma grande oportunidade de se alongar os ciclos de vida de elementos na antroposfera, reduzindo as necessidades de sua extração do meio ambiente. Isso é tanto mais necessário quando se considera que resíduos são, frequentemente, fontes de grandes problemas ambientais, mas poderiam ser soluções. ${ }^{15}$

O caso mais óbvio é o do lixo municipal, que causa uma enorme poluição nas cidades de todo o país, com raríssimas exceções. Este é um problema visível para quase todos, que não precisa ser descrito neste texto. Um jornal publicado em uma grande capital brasileira informou, em 26/5/2010, sobre um estudo realizado pela Associação Brasileira de Empresas de Limpeza Pública e Resíduos Especiais mostrando que mais de um terço (36\%) do lixo produzido pelos seus moradores era dirigido para o fundo de lagoas, rios e baías ou despejado em lixões clandestinos ou aterros irregulares. A estimativa, naquela data, era de 7.189 toneladas diárias de detritos, dos quais 879 toneladas não eram recolhidas de ruas e favelas.

O trato responsável de resíduos pode e deve utilizar diferentes estratégias: a eliminação de produtos e cadeias produtivas geradoras de resíduos intratáveis, a redução consciente de consumo, para reduzir o volume de resíduos produzidos em qualquer lugar e em qualquer tempo, a reciclagem, a gaseificação e/ou incineração para produzir energia térmica e elétrica, a compostagem geradora de fertilizantes e condicionadores de solos.

No Brasil, progressos no aproveitamento de resíduos têm sido lentos, seja pela falta de implementação de tecnologias bem comprovadas, seja pela falta de pesquisa e desenvolvimento necessários à solução de alguns problemas.

No caso da geração de energia, há publicações que abordam o problema de forma muito competente e exaustiva. Um exemplo é uma tese de doutorado defendida na UFRJ, produzida no seio de uma equipe experiente, ${ }^{16}$ que motivou a criação da empresa Usinaverde, operadora de uma planta de processamento de lixo, junto à UFRJ.

Essa tese informa que a produção de energia a partir do lixo pode responder por $30 \%$ da energia elétrica produzida no país, a custos comparáveis ou vantajosos face aos de outros processos de geração. Por isso mesmo é de estranhar que os seus ensinamentos não tenham influenciado fortemente as políticas públicas dessa área, como a política nacional de resíduos sólidos. ${ }^{17}$ Essa situação é explicada reconhecendo que existe na população um sentimento bastante disseminado de rejeição a processos de combustão, baseado na emissão de dioxinas e compostos furânicos em quantidades agressivas à saúde humana e ao ambiente.

As alegações da contaminação por dioxinas lembram que nos EUA, em 1987, os incineradores eram responsáveis pela geração de $82 \%$ das dioxinas no país, mas ignoram que em 2002, esse número caiu para apenas $3 \%$, sem que o total de emissões tenha aumentado significativamente. Na Grã-Bretanha, entre 1990 e 2000, as emissões de chumbo por incineradores caíram $97 \%$ e as de dioxinas caíram $99 \%$. Diversas usinas de incineração de lixo no Reino Unido, como a de Edmonton (Londres), emitem menos de 1/3 do limite brasileiro de dioxinas, portanto operam com tecnologias perfeitamente adequadas às exigências brasileiras. Um relatório ministerial da Alemanha ${ }^{18}$ informa que em 1990, 1/3 de todas as emissões de dioxinas na Alemanha 
foram produzidas por incineradores, mas em 2000 a contribuição dos incineradores estava reduzida a menos que 1\%. Em 2005, lareiras e chaminés residenciais emitiam aproximadamente 20 vezes mais dioxinas no ambiente do que as usinas de incineração. Portanto, as objeções à incineração ignoram o que foi conseguido pela inovação, embora continuem sendo repetidas no Brasil.

\section{PLATAFORMAS DE TRANSFORMAÇÃO SUSTENTÁVEL DE MATERIAIS}

\section{Nanotecnologia}

A inovação tem sido historicamente dependente de novos materiais, o que é exemplificado pela existência das Idades do Bronze, do Ferro, etc.. A criação e produção competitiva de materiais tem tido um papel decisivo no desenvolvimento tecnológico e econômico, bem como na consolidação de estruturas de poder nacional e econômico, em toda a história humana. O século XX foi pródigo em exemplos dessa afirmação, seja no caso dos plásticos e borrachas, dos semicondutores, de metais e cerâmicas.

Nas últimas décadas, a nanotecnologia vem demonstrando um grande número de possibilidades de construção de materiais fascinantes e dotados de conjuntos de propriedades até aqui desconhecidos, a partir da auto-organização de matérias-primas triviais em materiais nano-estruturados. A estruturação de materiais nas várias escalas de tamanho está permitindo a superação de muitos paradigmas bem estabelecidos mas que foram derivados das propriedades de substâncias puras ou isoladas. Dessa forma, estão sendo criados numerosos exemplos de grande impacto, como, por exemplo, no caso dos meta-materiais.

A nanotecnologia é transversal e várias nanotecnologias já estão presentes nas linhas de produção e nos produtos desenvolvidos em todos os setores produtivos, inclusive energia, saúde, farmácia, recursos hídricos, petroquímica, agronegócio, eletroeletrônica, química fina, defesa, aeroespacial, automobilística, agronegócio e serviços. O desafio atual da nanotecnologia brasileira é investir nos segmentos onde o país conta com uma indústria viável e competitiva, para que esta se mantenha como tal, e também nos segmentos pouco competitivos, para aumentar sua competitividade.

A pesquisa em novos materiais é muito ampla e diversificada. São muitas as classes de materiais que hoje despertam grandes expectativas, sendo que várias delas já estão representadas nos mercados globais. Por essa razão, a simples enumeração que coloque em destaque uma ou outra classe de materiais é pouco proveitosa. É preferível seguir o caminho oposto: identificar grandes problemas, metas e objetivos nacionais que possam receber contribuições do desenvolvimento científico e tecnológico nacional, associando-lhes em seguida soluções nanotecnológicas criadoras de novos materiais que possam ser utilizados na consecução dessas metas e objetivos, ou rotas de desenvolvimento nanotecnológico que possam gerar respostas para problemas atuais e emergentes. A intensa investigação em novos materiais é imprescindível para que o país produza novos produtos capazes de competir no mercado internacional. Por exemplo, na indústria têxtil e de calçados não só os processos de fabricação se tornam cada vez mais importantes para a modernização do setor, mas o uso de novos materiais poliméricos e nanocompósitos aumenta a qualidade de produtos e diminui o custo da produção.

Existe no país uma intensa atividade de pesquisa e desenvolvimento em nanotecnologia, executada em centros de pesquisa, universidades e empresas e que está sendo organizada e consolidada no ambiente do SisNano, ${ }^{19}$ corrigindo uma crônica desatenção de agências brasileiras de fomento para com essa área.

A título de ilustração, o Inomat (INCT de Materiais Complexos
Funcionais) e o Laboratório Nacional de Nanotecnologia do Centro Nacional de Pesquisa em Energia e Materiais têm atuado nos seguintes tipos de produtos e processos, motivados pela demanda de empresas ou por demandas estratégicas do governo brasileiro:

1) Novas tecnologias de união de metais por atrito. São técnicas revolucionárias, que permitem soldagem sem fundir metais e resolvendo casos considerados como impossíveis, até aqui. Essas tecnologias têm interesse nas indústrias de petróleo e gás, automobilística, de construção civil;

2) Materiais poliméricos complexos para a construção de tubulação "inteligente" para o transporte de petróleo, dos poços até a superfície;

3) Adesivos especiais para montagem metal-mecânica, especialmente para a indústria automobilística;

4) Revestimentos de cilindros, para a indústria de papel e celulose;

5) Materiais nanoestruturados obtidos de produtos da biomassa, especialmente de resíduos e de minerais abundantes, para aplicações de grande escala na construção civil e equipamentos de transportes;

6) Materiais nanoestruturados para as áreas de saúde, agropecuária e proteção ambiental;

7) Materiais para sensores e dispositivos para diagnóstico médico, veterinário e agroambiental;

8) Materiais para a geração e armazenagem de energia elétrica.

\section{Biotecnologia}

A biotecnologia oferece imensas oportunidades de inovação para a sustentabilidade, demonstradas pela vigorosa e inovadora cadeia produtora baseada na cana de açúcar. ${ }^{20} \mathrm{O}$ etanol, inicialmente derivado de material residual da produção de açúcar, tornou-se um produto industrial extremamente importante. Ambos, açúcar e álcool tornaram-se matérias-primas de muitos produtos químicos: butanol, polietileno, polibutadieno, ceras, solventes "verdes", ácido glutâmico, lisina, tensoativos, nanossílica, celulose, papel e polpa, celulose microcristalina, poli(ácido hidroxibutírico). A escala de produção de algumas dessas substâncias é impressionante: cerca de 500 mil toneladas de lisina são hoje produzidas com matéria-prima fornecida pelas usinas de açúcar e álcool, no estado de São Paulo. Vários outros produtos são esperados em um futuro próximo: ácidos graxos com menor grau de mistura que o dos produtos obtidos de óleos e gorduras, hidrocarbonetos, dimetilfurano, combustíveis derivados de álcoois superiores. Além disso, o $\mathrm{CO}_{2}$ abundantemente produzido nas dornas de fermentação, isento de contaminantes agressivos, pode ser usado como matéria-prima na produção de microalgas que por si mesmas têm um grande potencial na produção de energia e matérias-primas.

\section{INOVAÇÃO REQUER NOVA CIÊNCIA}

Muito da inovação necessária para se conseguir sustentabilidade nas atividades humanas independe de novas tecnologias ou mesmo de nova ciência: qualquer pessoa pode contribuir para a redução de consumo predatório, mudando alguns hábitos e fazendo o aproveitamento cuidadoso de materiais e energia, seja no plano pessoal ou nas suas atividades profissionais, utilizando apenas práticas e tecnologias bem conhecidas e comprovadas.

Entretanto, também temos necessidade de muita nova ciência. A química tem tido um papel especialmente produtivo na transformação da matéria e na compreensão dos fenômenos envolvidos, mas muitas pessoas consideram que ela atingiu um estágio de maturidade do qual se espera poucas quebras de paradigmas e poucas grandes novidades. Isso não é verdade e a prática dos autores desse texto tem mostrado uma forte componente química em fenômenos considerados "físicos", 
como a eletrização de isolantes ${ }^{21} \mathrm{e} o$ atrito, ${ }^{22}$ que até recentemente foi ignorada.

\section{O CONTEXTO BRASILEIRO}

O Brasil desfruta hoje de uma posição muito singular e confortável: está se tornando um dos maiores produtores de petróleo e gás do mundo, ao mesmo tempo em que lidera a produção global de commodities agrícolas, importantes fontes de alimentos e energia. A crescente exploração de petróleo e gás está demandando investimentos muito expressivos atraindo grandes grupos internacionais. Da mesma forma a produção de commodities agrícolas atrai também grandes investimentos do exterior, frequentemente usados na aquisição de ativos de empresas nacionais com liderança tecnológica global.

Um trabalho anterior do autor demonstra a existência de uma grande sinergia na produção de alimentos, energia e também matérias-primas, ${ }^{7}$ quando feita de forma consciente, bem informada pelo estado da arte da ciência e tecnologia, sujeita a critérios éticos e a uma legislação aplicada competentemente. Já são muitos, no Brasil, os casos positivos de impacto favorável da atividade agrícola sobre o meio-ambiente, seja através do uso consciente do espaço, seja através da eliminação da miséria humana, que é causa de imensas agressões ambientais.

Uma questão que surge frequentemente é a do uso de terra para a produção de energia e matérias-primas, que supostamente impede o seu uso na produção de alimentos. Há bons exemplos sobre a impertinência dessas alegações e um deles é o fato de $1 / 10$ de todo o bagaço de cana produzido no Brasil ser utilizado em alimentação animal. São 17 milhões de toneladas de alimento por ano, obtidos de um subproduto das usinas.

\section{PROPOSTAS}

O aproveitamento inovador de biomassa, especialmente dos resíduos da produção de alimentos e de outras atividades humanas, bem como de minerais abundantes, na produção de materiais utilizados em diferentes setores da economia, é um caminho para se aumentar a sustentabilidade e evolução de atividades humanas, reduzindo ou eliminando dependências de matérias-primas cujo esgotamento é previsível.

Esse aproveitamento pode e deve ser feito utilizando a melhor ciência e engenharia disponíveis, viabilizando inovações que tragam resultados econômicos, sociais e estratégicos que, por si mesmos, garantam a sustentabilidade das empresas envolvidas e recebam o apoio do público e de governos, que por sua vez garanta a sustentabilidade das próprias atividades de $\mathrm{P} \& \mathrm{D}$.

Uma importante batalha a ser vencida nos processos de inovação é a necessidade de se desenvolver materiais que tenham baixo risco e não tragam impactos adversos sobre a saúde humana e o meio ambiente. É preciso pesquisar e compreender casos como o das nanopartículas de óxidos, que tem mostrado grande complexidade na ação biológica. ${ }^{23} \mathrm{O}$ risco está sempre presente no desenvolvimento e adequação de qualquer material, por mais que seja chamado de verde ou avançado. Portanto, é imprescindível que seja feita a análise de risco de um novo material antes de sua fabricação e aplicação, evitando vultosas perdas econômicas, ambientais ou humanas e as consequentes reações adversas do público.

\section{AGRADECIMENTOS}

O autor agradece aos seus estudantes, pós-graduandos e outros colaboradores, pelo sua contribuição à consolidação das análises e propostas apresentadas neste trabalho e também ao CNPq e Fapesp pelo apoio ao Inomat, INCT\&Inovação de Materiais Complexos Funcionais.

\section{REFERÊNCIAS}

1. Pinto, A. C.; Zucco, C.; Galembeck, F.; Andrade, J. B.; Vieira, P. C.; Quim. Nova 2012, 35, 2092.

2. http://www.clubofrome.org/?p=375, acessada em Julho 2013.

3. www.dnpm.gov.br/assets/galeriaDocumento/SumarioMineral2008/ fosfato.pdf, acessada em Julho 2013.

4. http://blogs.ei.columbia.edu/2013/04/01/phosphorus-essential-to-lifeare-we-running-out/, acessado em Julho 2013.

5. Fletcher, T.; Scott, J. L.; Materials for a Sustainable Future, Royal Society of Chemistry: Londres, 2012.

6. Galembeck, F.; Csordas, Y.; Em Materiais Avançados 2010-2012; Rizzo F.C; Medeiros E.F.; Fellows L., eds.; CGEE: Brasília, 2009, cap. 6.

7. Galembeck, F.; Energy Environ. Sci. 2010, 3, 393.

8. Galembeck, F.; Barbosa, C. A. S. ; Sousa, R. A.; Quim. Nova 2009, 32, 571.

9. Galembeck, F.; Rippel, M. M.; Revista Parcerias Estratégicas 2004, 18, 41; Alexandre, M.; Dubois, P.; Mater. Sci. Eng., $R$ 2000, 28, 1; Giannelis, E. P.; Adv. Mater. 1996, 8, 29; LeBaron, P. C.; Wang, Z.; Pinnavaia, T. J.; Appl. Clay Sci. 1999, 15, 11.

10. Beg, M. D. H.; Pickering, K. L.; Polym. Degrad. Stab. 2008, 93, 1939; Plackett, D.; Andersen, T. L.; Pedersen, W. B.; Nielsen, L.; Compos. Sci. Technol. 2003, 63, 1287.

11. Gouveia, R. F.; Galembeck, F.; J. Am. Chem. Soc. 2009, 131, 11381; Valadares, L. F.; Braganca, F. D. C. ; da Silva, C. A. ; Leite, C. A. P.; Galembeck, F.; J. Colloid Interface Sci. 2007, 309, 140; Rosseto, R.; dos Santos, A. C. M. A. ; Galembeck, F.; J. Braz. Chem. Soc. 2006, 17, 1465; Beppu, M. M.; Lima, E. C. D. O.; Galembeck, F.; J. Colloid Interface Sci. 1996, 178, 93; Galembeck, F.; De Brito, J.; Santos, A. C. M. A.; Rosseto, R.; US pat. 200800385562008.

12. Rippel, M. M. ; Linares, E. M. ; Braganca, F. C. ; Valadares, L. F. ; Galembeck, F.; J. Adhes. Sci. Technol. 2012, 26, 767; Linares; E. M.; Jannuzzi, S. A. V.; Galembeck, F.; Langmuir 2012, 27, 15199; Rezende, C. A.; Bragança, F. C.; Doi, T. R.; Lee, L. T.; Galembeck, F.; Boué, F.; Polymer 2010, 51, 3644; Linares, E. M.; Rippel, M. M.; Galembeck, F.; ACS Appl. Mater. Interfaces 2010, 2, 3648; Valadares L. F.; Leite, C. A. P.; Galembeck, F.; Polymer 2006, 47, 672; Valadares, L. F.; Murakami, M. M.; Rippel, M. M.; Galembeck, F.; Br PI 0301193-3, 2003.

13. Linares, E. M.; Leite, C. A. P.; Valadares, L. F.; Silva, C. A.; Rezende, C. A.; Galembeck, F.; Anal. Chem. 2009, 81, 2317.

14. Rezende, C. A.; Lima, M. A.; Maziero, P.; de Azevedo, E. R.; Garcia, W.; Polikarpov, I.; Biotechnol. Biofuels 2011, 4, 1; Gurgel, L. V. A.; Marabezi, K.; Zambom, M. D.; Curvelo, A. A. S.; Ind. Eng. Chem. Res. 2012, 51, 1173 .

15. http://www.jornaldaciencia.org.br/Detalhe.jsp?id=88300, acessado em Julho 2013

16. Oliveira, L. B.; Tese de Doutorado, Universidade Federal do Rio de Janeiro, Brasil, 2004.

17. http://www.planalto.gov.br/ccivil_03/_ato2007-2010/2010/lei/112305. htm, acessado em Julho 2013.

18. http://www.seas.columbia.edu/earth/wtert/sofos/Waste_Incineration_A_ Potential_Danger.pdf, acessado em Julho 2013.

19. www.mct.gov.br/sisnano, acessada em Julho 2013

20. Macedo, I. C.; A Energia da Cana de Açúcar, Berlendis \& VertecchiaÚnica: São Paulo, 2005.

21. Burgo, T. A. L; Ducati, T. R. D.; Francisco, K. R.; Clinckspoor, K. J.; Galembeck, F.; Galembeck, S. E.; Langmuir 2012, 28, 7407.

22. Burgo, T. A. L.; Silva, C. A.; Balestrin, L. B. S.; Galembeck, F.; Sci. Rep., no prelo.

23. Xia, T.; Kovochich, M.; Liong, M.; Mädler, L.; Gilbert, B.; Shi, H.; Yeh, J. I.; Zink, J. Y.; Nel, A.E.; ACS Nano 2008, 2, 2121. 